\title{
Environmental management zoning for coal mining in mainland China based on ecological and resources conditions
}

\author{
Haiqing Geng • Fan Chen • Zhiyuan Wang • Jie Liu • \\ Weihua Xu
}

Received: 29 June 2016 / Accepted: 7 April 2017 /Published online: 22 April 2017

(C) Springer International Publishing Switzerland 2017

\begin{abstract}
The purpose of this research is to establish an environmental management zoning for coal mining industry which is served as a basis for making environmental management policies. Based on the specific impacts of coal mining and regional characteristics of environment and resources, the ecological impact, water resources impact, and arable land impact are chose as the zoning indexes to construct the index system. The ecological sensitivity is graded into three levels of low, medium, and high according to analytical hierarchy processes and gray fixed weight clustering analysis, and the water resources sensitivity is divided into five levels of lower, low, medium, high, and higher according to the weighted sum of sub-indexes, while only the arable land sensitive zone was extracted on the basis of the ratio of arable land to the county or city. By combining the ecological sensitivity zoning and the water resources sensitive zoning and then overlapping the
\end{abstract}

\footnotetext{
H. Geng · F. Chen · J. Liu

Appraisal Center for Environment and Engineering, Ministry of Environmental Protection, Beijing 100012, People's Republic of China

\section{Z. Wang $(\bowtie)$}

Marine Environmental Engineering Center, South China Sea Institute of Oceanology, Chinese Academy of Sciences, 164 West Xingang Road, Guangzhou 510301, People's Republic of China e-mail: zwang@scsio.ac.cn
}

W. Xu

Research Center for Eco-environmental Sciences, Chinese Academy of Sciences, Beijing 100085, People's Republic of China arable-sensitive areas, the mainland China is classified into six types of environmental management zones for coal mining except to the forbidden exploitation areas.

Keywords Zoning · Environmental management $\cdot$ Coal mining

\section{Introduction}

Coal mining is one of the largest natural exploitation activities in mainland China, and the coal accounts for about $70 \%$ of China's total primary energy production and consumption. In 2010, about 3.24 and 3.12 billion tons of coal were produced and consumed, respectively, which accounted for 48.3 and $48.2 \%$ of the total in the world (National Bureau of Statistics of China 2012). Unfortunately, the coal mining is also one of the largest eco-damaging activities which has the potential to destroy flora and fauna, breach groundwater system and contaminate soil, air, and water in the surrounding areas (Driussi and Jansz 2006; Li and Zhou 2006; Si et al. 2010). For example, $63,000 \mathrm{hm}^{2}$ of subsidence area and $69,000 \mathrm{hm}^{2}$ of erosion area were caused by coal mining in 2010. In addition, $61 \times 10^{8} \mathrm{~m}^{3}$ waste water and $5.94 \times 10^{8}$ tons of coal slack has been discharged. ${ }^{1}$ Either the earth subsidence or the pollutant emission can lead to serious environmental problems. Owing to the fast industrialization and urbanization, the

\footnotetext{
${ }^{1}$ Shenhua Research Institute. Study on the Standard and Policy of Ecological Civilization Mining, 2015.
} 
development of coal mining industry has been accelerated since 2000, which would impose enormous stress on the natural environment. The Appraisal Center for Environment and Engineering (ACEE), MEP, P. R. China) has reviewed 180 EIA reports of large proposed coal mines during 2001-2008, which the total scale of production was up to 875 million tons and the investment exceeded the sum of that in past 50 years (Geng et al. 2010). Therefore, it is urgent to strengthen the environmental management for coal mining industry.

Zoning in essence is a behavior of dividing a region into different sections based on its natural, economic, or social attributes. National or local governments can achieve their macro policy objectives such as optimizing the industrial layout, balancing the development among different regions, and improving the efficiency of resource allocation by making distinctive policies towards different areas based on the formulated zonings. There are two spatial plans for coal mining industry in mainland China. One was issued in the 1980s, in which the whole mainland China is divided into three planning zones as Eastern coal importing zone, Central coal exporting zone, and the Western coal reserving zone. The three zones are further divided into seven planning areas based on the regional coal resource condition, coal production, consumption, and transportation. The other was enacted in 2006 called the National Large Coal Bases Planning, which divided the coal-rich regions into 13 large coal bases consisting of 98 national planning coal districts. The functions, development goals, and detailed developing plans for each base were put forward in the plan. The major tasks of both the above plans, however, were to promote the efficiency of resource allocation among different regions, while resource and environmental carrying capacity were less considered. With the continuous degradation of the ecological environment attributed to human activities, social, economic, demographic, resources and environmental factors, etc., have become more and more important in zoning (Dwyer et al. 2004; Sun 2010). So, it is necessary to explore environmental management zonings for coal industry according to their significant resources and environmental impacts and the corresponding carrying capacity in different regions, based upon which more detailed and targeted environmental management policies can be formulated. Therefore, we raise a concept of environmental management zone and divide mainland China into several environmental management zones for coal mining industry according to its main environmental impacts and the corresponding regional characteristics.

\section{Methods}

Indexes selection and zoning processes

The negative environmental impacts of coal mining mainly include (1) ecological damage and landscape alteration due to the earth surface subsidence, land damage, land occupation, and the construction of infrastructures; (2) physical damage on the regional hydrology and the stress on local water supply arose by the water consumption of coal mining and its downstream industries; (3) the reduction of arable land resulted from the occupation or subsidence of coal mining; and (4) the environmental pollutions caused by emissions of wastes during coal mining. Compared to ecological impacts, water resource damage, and the arable land destruction, the environmental pollution from coal mining is not serious in China, so only the ecological impact, water resource impact, and arable land impact are taken as the zoning indexes in the present paper.

Figure 1 schematically illustrates the process of zoning. As shown in the figure, the step of zoning proceeded as follows: constructing an index system of

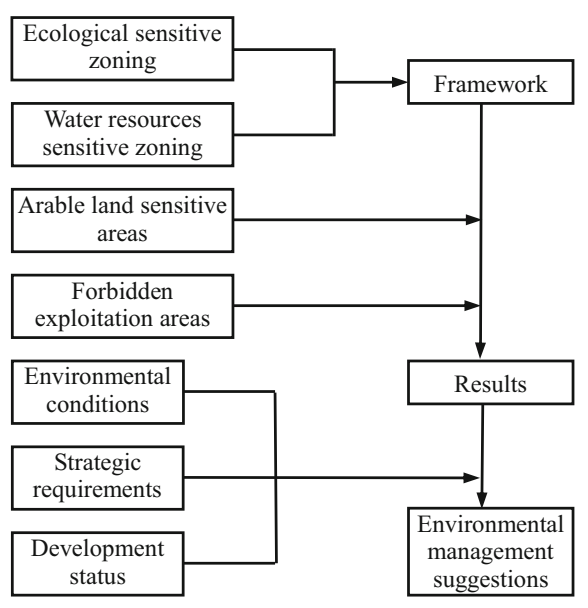

Fig. 1 Technique route of the zoning 
ecological, water resource, and arable land sensitivities; conducting an ecological sensitivity and a water resource sensitivity division, respectively; forming a basic zoning framework by combining the ecological sensitivity and the water resource divisions; getting the final zoning by overlapping arable land-sensitive and forbidden exploitation areas on the basic zoning framework; and putting forward some strategic suggestions and solutions in the light of each type of zone.

\section{Index system construction}

Different evaluation indexes of coal industry environments and ecological evaluation models of mining sites have been studied (Song et al. 2007; Tyra et al. 2003; Xue et al. 2006; Zhang et al. 2013) based on different purposes. For example, Si et al. (2010) put forward an evaluation index system of environmental sustainability in coal mining based on the aim of recycle economy and a method of sustainability evaluation. In this study, the following four principles should be followed in selecting the sub-indexes and data: (1) each sub-index should be representative and can capture one specific character of the upper index, (2) the information supplied by each different sub-index should be avoided overlapping as much as possible, (3) the data selected should be systematic and reliable, and (4) the data should be spatial auto correlative and can reflect a regional differentiation for ease of translating the zoning results-specific management policy. Thus, the four sub-indexes of soil erosion sensitivity $\left(B_{1}\right)$, desertification sensitivity $\left(B_{2}\right)$, rocky desertification sensitivity $\left(B_{3}\right)$, and biological diversity $\left(B_{4}\right)$ are chosen to construct the ecological sensitivity $\left(A_{1}\right)$ which reflects the ecological impact caused by coal mining, the two specific sub-indices of regional water abundance $\left(B_{5}\right)$ and rainfall $\left(B_{6}\right)$ are selected to construct the water resource sensitivity $\left(A_{2}\right)$ which reflect the abundance of water resources, and the arable land density $\left(B_{7}\right)$ is used to represent the arable land sensitivity $\left(A_{3}\right)$.

The evaluation index system and the weight coefficients of the indexes are presented in Table $1 . B_{1}, B_{2}$, and $B_{3}$ are denoted by the classification data $\left(C_{1}, C_{2}, C_{3}\right.$, respectively) with the same meaning, and $B_{4}$ is denoted by the quantity of indicator species $\left(C_{4}\right) . C_{1}, C_{2}, C_{3}$, and $C_{4}$ were transferred from the corresponding raster data of 2008 from the Chinese Academy of Sciences. $B_{5}$ and $B_{6}$ are denoted by the available water resources per square kilometer $\left(C_{5}\right)$ and the average annual rainfall $\left(C_{6}\right)$, respectively. $C_{5}$ and $C_{6}$ were the survey and statistical data of 2010 from the China Institute of Water Resources and Hydropower Research. $B_{7}$ is denoted by the ratio of cultivatable land to total land at a county or city level $\left(C_{7}\right)$, the data of $C_{7}$ were cited from the 2011 Statistical Yearbook (published by the National Bureau of Statistics of China).

The weights of $C_{1}-C_{4}$ in $A_{1}$ are determined by the analytical hierarchy processes (Svoray and Ben-Said 2010) and are $0.5638,0.2634,0.0550$, and 0.1178 , respectively. In summary, a relatively judgment matrix for $C_{1}-C_{4}$

Table 1 Indices, sub-indexes, data and the corresponding weights used in zoning

\begin{tabular}{llll}
\hline Indexes & Sub-indexes & Data & Weights \\
\hline Ecological sensitivity $\left(A_{1}\right)$ & Soil erosion sensitivity $\left(B_{1}\right)$ & $\begin{array}{l}\text { Classification of soil erosion } \\
\text { sensitivity }\left(C_{1}\right)\end{array}$ & $\begin{array}{l}\text { Classification of desertification } \\
\text { sensitivity }\left(C_{2}\right)\end{array}$ \\
& Desertification sensitivity $\left(B_{2}\right)$ & $\begin{array}{l}\text { Classification of rocky desertification } \\
\text { sensitivity }\left(C_{3}\right)\end{array}$ & 0.5638 \\
& Rocky desertification & Quantity of indicator species $\left(C_{4}\right)$ & 0.0550 \\
Sensitivity $\left(B_{3}\right)$ & Available water resources per square & kilometer $\left(C_{5}\right)$ \\
Water resources sensitivity $\left(A_{2}\right)$ & Biological diversity $\left(B_{4}\right)$ & Average annual rainfall $\left(C_{6}\right)$ & 0.1178 \\
& Abundance of water resources $\left(B_{5}\right)$ & Ratio of arable land $\left(C_{7}\right)$ & 0.3234 \\
Arable land & Rainfall $\left(B_{6}\right)$ & & 1.0000 \\
sensitivity $\left(A_{3}\right)$ & Arable land density $\left(B_{7}\right)$ & & \\
\hline
\end{tabular}


Table 2 Comparison matrix for $\mathrm{C}_{1}-\mathrm{C}_{4}$

\begin{tabular}{lllll}
\hline & $\mathrm{C}_{1}$ & $\mathrm{C}_{2}$ & $\mathrm{C}_{3}$ & $\mathrm{C}_{4}$ \\
\hline $\mathrm{C}_{1}$ & 1 & 3 & 7 & 5 \\
$\mathrm{C}_{2}$ & $1 / 3$ & 1 & 5 & 3 \\
$\mathrm{C}_{3}$ & $1 / 7$ & $1 / 5$ & 1 & $1 / 3$ \\
$\mathrm{C}_{4}$ & $1 / 5$ & $1 / 3$ & 3 & 1 \\
\hline
\end{tabular}

was firstly determined by experts, and then the weights of $C_{1}-C_{4}$ were calculated with the following two expressions:

$$
\begin{aligned}
& \varpi_{i}=\left(\prod_{j=1}^{4} C_{i j}\right)^{1 / 4}, i=1,2,3,4 \\
& \omega_{i}=\varpi_{i} / \sum_{k=1}^{4} \varpi_{k}, i=1,2,3,4
\end{aligned}
$$

where $\varpi_{i}$ is the geometric mean of the sum of all the elements in each row of the matrix as showed in Table 2, and $\omega_{i}$ is the weight of sub-index.

The weights of $C_{5}$ and $C_{6}$ in $A_{2}$ are determined by Delphi (Wang and Si 2011) and are 0.5766 and 0.4234, respectively. The weight of $C_{7}$ is 1 because $C_{7}$ is the only indicator of $A_{3}$.

Data processes

The data analysis was conducted on the platform of ArcGIS 10 and the data were calculated at the county or city level. Figure 2 schematically shows the data processing and indexes calculation procedure. First, all of the retrieved data were turned into vector data, and then assigned property values to establish a fundamental spatial database by the ways of analysis calculation, direct reference, or subjective valuation, respectively. On the basis, the three indexes of ecological, water resources, and arable land sensitivities were calculated, and then the final zoning were constructed according to the zoning procedure showed in Fig. 1.

The ecological sensitivity is classified into 3 Gy types of low, medium, and high, and their values are calculated through gray fixed weight clustering analysis (Moran et al. 2006). The whitenization weight function of gray type $k$ to sub-index $j f_{j}^{k}(x)$ $(k=1,2,3 ; j=1,2,3,4)$ is established according to the consultation from experts (where the $k$ of 1,2, and 3 represent the gray types of "low," "medium," and "high," respectively, and the $j$ of $1,2,3$, and 4 represent the sub-index of $B_{1}, B_{2}$, $B_{3}$, and $B_{4}$, respectively). The whitenization functions of $B_{1}, B_{2}$, and $B_{3}$ are discrete due to $B_{1}, B_{2}$, and $B_{3}$ are classification data, while that of $B_{4}$ is continuous because of $B_{4}$ being denoted by the quantity of indicator species.

The three whitenization weight functions for soil erosion sensitivity are as follows:

$f_{1}^{1}(x)=\left\{\begin{array}{cc}1 & x=1 \\ 0 & x \geq 2\end{array} f_{1}^{2}(x)=\left\{\begin{array}{cc}0 & x=1 \\ 0.5 & x=2 \\ 1 & x=3 \\ 0 & x \geq 4\end{array} f_{1}^{3}(x)=\left\{\begin{array}{cc}0 & x \leq 3 \\ 1 & x \geq 4\end{array}\right.\right.\right.$

The three whitenization functions for desertification sensitivity are as follows:

Fig. 2 Data processing and indexes calculation procedure

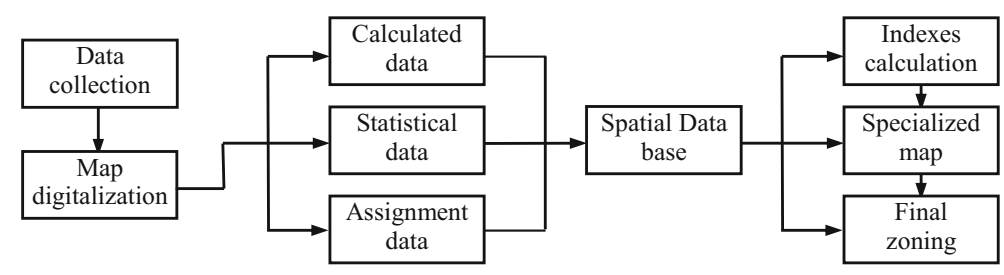


$f_{2}^{1}(x)=\left\{\begin{array}{cc}1 & x=1 \\ 0 & x \geq 2\end{array} \quad f_{2}^{2}(x)=\left\{\begin{array}{cc}0 & x=1 \\ 1 & x=2 \\ 0.5 & x=3 \\ 0 & x \geq 4\end{array} \quad f_{2}^{3}(x)=\left\{\begin{array}{cc}0 & x \leq 2 \\ 0.5 & x=3 \\ 1 & x \geq 4\end{array}\right.\right.\right.$

The three whitenization weight functions for rocky desertification sensitivity are as follows

:

$f_{3}^{1}(x)=\left\{\begin{array}{cc}1 & x=1 \\ 0 & x \geq 2\end{array} \quad f_{3}^{2}(x)=\left\{\begin{array}{cc}0 & x=1 \\ 1 & x=2 \\ 0 & x \geq 3\end{array} \quad f_{3}^{3}(x)=\left\{\begin{array}{cc}0 & x \leq 2 \\ 0.5 & x=3 \\ 1 & x \geq 4\end{array}\right.\right.\right.$

The three whitenization weight functions for quantity of indicator species are as follows:

$f_{4}^{1}(x)=\left\{\begin{array}{cc}1 & x \leq 10 \\ \frac{30-x}{20} & 10<x<30 \\ 0 & x \geq 30\end{array} \quad f_{4}^{2}(x)=\left\{\begin{array}{cc}0 & x \leq 10 \\ \frac{x-10}{20} & 10<x \leq 30 \\ 50-x & 30<x \leq 50 \\ \frac{50}{20} & x>50\end{array} \quad f_{4}^{3}(x)=\left\{\begin{array}{cc}0 & x \leq 30 \\ \frac{x-30}{20} & 30<x \leq 50 \\ 1 & x>50\end{array}\right.\right.\right.$

According to the weights from the analytical hierarchy processes, the clustering coefficient of gray class $k$ for object $i\left(\sigma_{i}^{k}\right)$ can be given by:

$\sigma_{i}^{k}=\sum_{j=1}^{m} f_{j}^{k}\left(x_{i j}\right) \omega_{j} \quad j=1,2,3,4 ; k=1,2,3$

where $f_{j}^{k}\left(x_{i j}\right)$ is the whitenization weight function and $\omega_{j}$ is the weight of sub-index. If $\max _{1 \leq k \leq 3}\left\{\sigma_{i}^{k}\right\}=\sigma_{i}^{k^{*}}$, the object $i$ belongs to the gray class $k^{*}$.

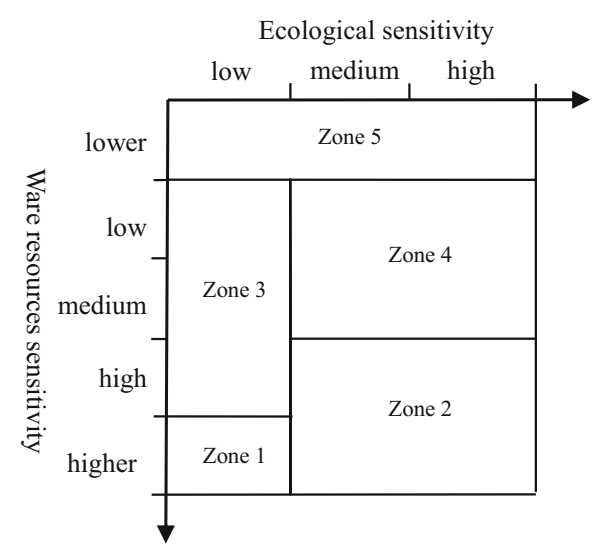

Fig. 3 Framework from combination of ecological sensitivity and water resources sensitivity 
The water resource sensitivity $\left(A_{2}\right)$ is classified into five grades of "lower," "low," "medium," "high," and "higher" according to the Quantile in ArcGIS, and its value is calculated by weighting sum of available water resources per square kilometer $\left(C_{5}\right)$ and annual average rainfall $\left(C_{6}\right)$. It is assumed that the smaller value implies more sensitivity to the coal mining. The differences of $C_{5}$ and $C_{6}$ in dimension and magnitude are eliminated by the following normalization formula:

$$
x_{i j}^{*}=\frac{x_{i j}-\min \left(x_{j}\right)}{R\left(x_{i j}\right)}=\frac{x_{i j}-\min \left(x_{j}\right)}{\max \left(x_{j}\right)-\min \left(x_{j}\right)}
$$

The arable land sensitive index $\left(A_{3}\right)$ is calculated by the ratio of arable land $\left(C_{7}\right)$ at the county or city level.

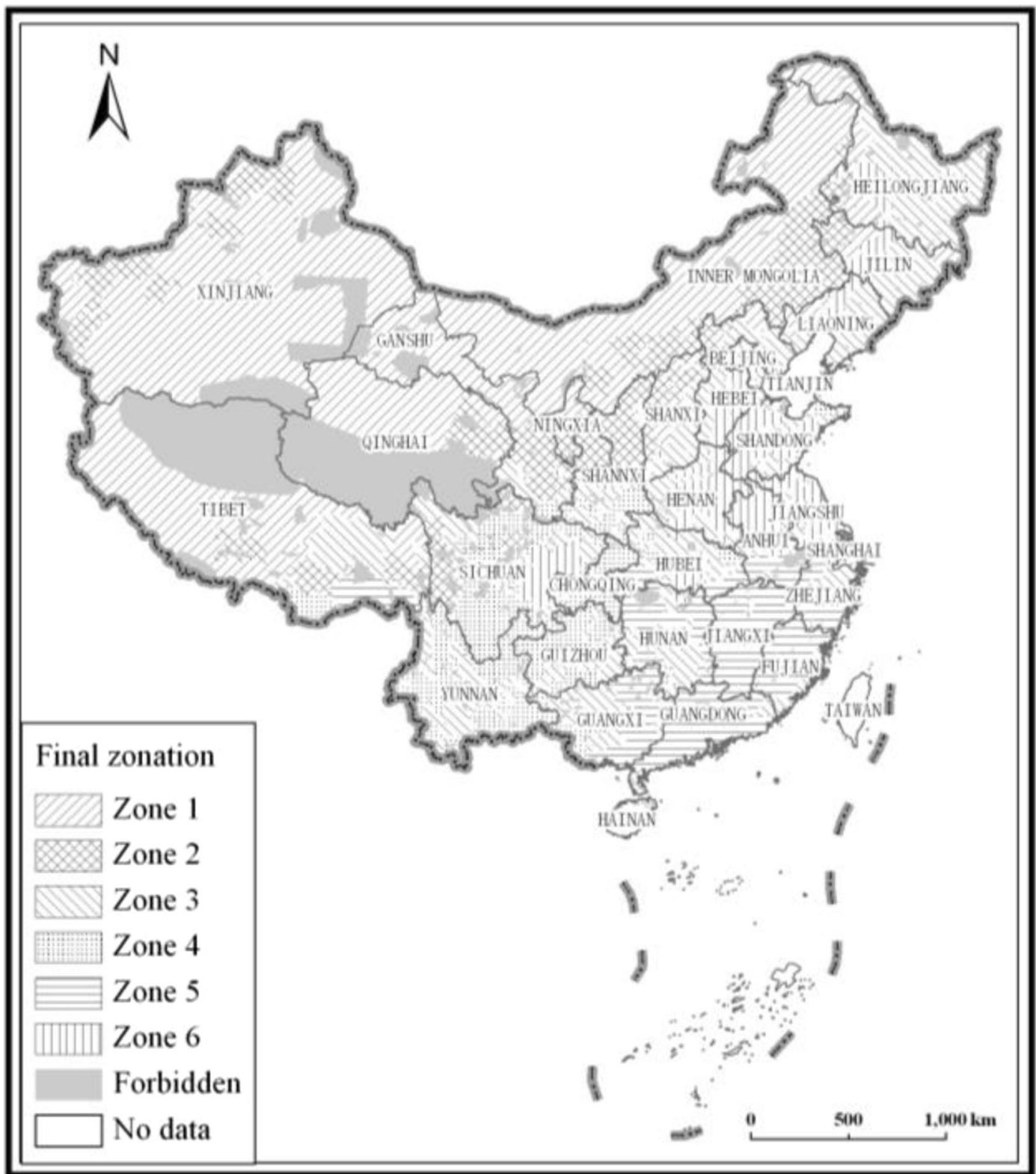

Fig. 4 Zoning map for environmental management of coal mining in mainland China 


\section{Results and discussion}

The framework of environmental management zones for coal mining is basically determined by its ecological impact and water resource impact because they are the two dominant impacts. Another reason is that both the ecosystem and water resources have obvious zonal structure in China. Theoretically, there are 15 types of zones according to the combination of previous ecological sensitivity zoning and water resource sensitivity zoning as ecological sensitivity has three classifications and water resource sensitivity has five classifications. In present paper, the contiguous zones with similar ecological and hydrological characteristics are merged to form the framework of environmental management zones including five primary types of zones on which the arable land sensitive areas are overlapped (Fig. 3). In addition, the forbidden exploitation areas such as nature reserves, scenic spots, forest parks, drinking water source protection areas, and cultural heritage areas are excluded from the environmental management zones in order to avoid ambiguity. Thus, the mainland China is divided into six types of environmental management zones for coal mining as shown in Fig. 4. Table 3 lists the spatial characteristics of the six zones, and it is found that areas of zones $1-6$ occupy $45.8,12.6,17.8,8.4,8.9$, and $6.5 \%$ of the total land area of mainland China, respectively.

The typical landscape of zone 1 is plateau, which is mainly located in the northern Inner Mongolia, Northwestern Gansu, most parts of Xinjiang, Tibet, and Qinghai, where the soil erosion and desertification are serious due to the arid climate and the irrational natural resources exploitation. The coal resources in zone 1 are quite suitable for large-scale mechanized mining because the coal bed is usually thick and the geological structure is simple. The most existing coal mines are concentrated in Inner Mongolia and Xinjiang with large or medium size. In recent years, the exploiting intensity of coal mine has been enhanced because of the increasing demand of energy. Water resource shortage is the main restricting factor for the coal mining and its downstream industries; therefore, how to efficiently allocate and utilize the water resources should be considered in priority in mining exploitation activities.

Zone 2 are mainly distributed in the transition zone between China's first and second ladder terrains, where the geomorphic type is mainly hilly area, such as the contiguous areas of Shanxi, Shaanxi, and Inner Mongolia and the southern Ningxia and Gansu, western Xinjiang, part of Eastern Inner Mongolia such as Chifeng, Tongliao, and Hinggan League. In the regions, the coal reserves are extremely rich and account for more than $60 \%$ of the total in mainland China, but the serious soil erosion, land desertification, and water resources scarcity have restricted the regional development. Because of the excellent resource conditions, most of the existing, constructing, and planning projects are large coal mines, and the contiguous areas of Shanxi, Shaanxi, and Inner Mongolia have already become an important national energy and heavy chemical base of China. However, the shortage of water resources in the region can hardly support the higher-intensity exploitation again, and the regions have been suffered serious environmental pollution caused by the large quantity of thermal power plants and heavy chemical industries.

Zone 3 regions are mainly distributed in the eastern Northeast China Plain, western North China Plain, the contiguous areas of Sichuan, Gansu, and Qinghai, and some areas in central and eastern China. Most of these regions are hilly areas or the transition zones between hilly areas and plains. The coal resources are mainly

Table 3 Spatial distribution of zones 1-6 in mainland China

\begin{tabular}{|c|c|c|c|}
\hline Zones & Regions & No of units & $\begin{array}{l}\text { Percentage of } \\
\text { land area }(\%)\end{array}$ \\
\hline Zone 1 & Northern Inner Mongolia, North-western Gansu, most parts of Xinjiang, Tibet and Qinghai & 245 & 45.8 \\
\hline Zone 2 & $\begin{array}{l}\text { Contiguous areas of Shanxi, Shaanxi and Inner Mongolia, southern Ningxia and Gansu, } \\
\text { western Xinjiang, part of Eastern Inner Mongolia }\end{array}$ & 263 & 12.6 \\
\hline Zone 3 & $\begin{array}{l}\text { Eastern Northeast China Plain, western North China Plain, contiguous areas of Sichuan, } \\
\text { Gansu and Qinghai, parts in Guangxi, Guizhou, etc. }\end{array}$ & 661 & 17.8 \\
\hline Zone 4 & Most parts of Sichuan, Chongqing, Guizhou, and Yunnan & 291 & 8.4 \\
\hline Zone 5 & Jiangxi, Zhejiang, Fujian, Guangdong, and Hainan; parts of Hunan, Guangxi, and Anhui & 450 & 8.9 \\
\hline Zone 6 & North China Plain, Songnen Plain, and Sichuan Basin & 468 & 6.5 \\
\hline
\end{tabular}


concentrated in Heilongjiang, Liaoning, Shanxi, and north of Henan with deep buried bed and unstable geological structure, and the most of existing coal mines are large scale. However, the coal resource exhaustion, the surface subsidence, land damage, and environmental pollution are rather serious due to the long history of coal mining.

Zone 4 regions are located in the ecologically fragile areas in south-western China and include most parts of Sichuan, Chongqing, Guizhou, and Yunnan with complicated topography and rich biodiversity. The zone 4 regions have typically karst terrain with serious rocky desertification, but abundant ground and surface water. Coal resources in the regions are mainly concentrated in the contiguous areas of Guizhou, Yunnan, and Sichuan, and most of the existing coal mines are medium or small scale. The coal mining in the regions is mainly subject to the following problems: (1) the complicated geological condition as well as the high content of sulfur and gas in coal beds make the exploitation cost much higher than other regions; (2) the downstream industries such as power and coal chemistry will be bound to increase sulfur dioxide pollution significantly due to the high sulfur content in coal; (3) the content of arsenic in coal bed of some areas is also high, which would lead to significant environmental hazards; (4) the geological disaster caused by subsidence is very serious and should be concerned; and (5) the coal mining is imposing great threaten to the local ecosystems.

Zone 5 regions are mainly located in the southeastern China, including Jiangxi, Zhejiang, Fujian, Guangdong, Hainan, and parts of Hunan, Guangxi, and Anhui, where the primitive natural ecosystem is quite rare owing to the long history of agricultural development and the relatively high level of development. The coal mines are usually small-scale and low yield due to the poor resource conditions; therefore, the ecological damage and environmental pollutions caused by coal mining are not so prominent. But, compared with its environmental impacts, the contribution of coal mining to local economy is even negligible.

Zone 6 regions are located in the main grainproducing areas such as North China Plain, Songnen Plain, and Sichuan Basin. The coal resources are mainly distributed in the contiguous areas of Henan, Anhui, and Shandong with the deep and thick coal beds, and most of the existing coalmines are large or medium scaled. Although the coal mining and its downstream industries have developed very fast since 2000 , the current development mode faces some serious constraints which are as follows: (1) the coal reserves keep decline and can no longer support the high intensity of exploitation; (2) more than 50\% of arable land would lose the farming function due to subsidence, which conflicts with the strict policy of arable land protection in China; (3) more than 10,000 people will be usually resettled just for constructing one coal mine to avoid suffering subsidence, and the cost is too much to endure; and (4) the impacts of the environmental pollution to people caused by coal mining is also serious owing to the high density of population.

\section{Conclusions}

The current environmental management system of mainland China has not given full considerations to the specific impacts of different industries and regional characteristics of environment and resources. Therefore, we proposed a new concept of environmental management zoning in the paper and have divided the mainland China into six broad categories of zones for coal mining based on the resources and ecological conditions of regions and the main environmental impacts of coal mining. It should be noted that the present study is just an exploration for zoning of environmental management and both the theory and method are developing. Other more comprehensive factors, such as national energy security, transportation, and regional development status, should be incorporated to the model and a more integrated environmental management zoning should be conducted in the further study.

Acknowledgements The financial was supported by the Ministry of Environment Protection, P.R. China with a grand no. 200809072 .

\section{References}

Driussi, C., \& Jansz, J. (2006). Pollution minimisation practices in the Australian mining and mineral processing industries. Journal of Cleaner Production, 14, 673-681.

Dwyer, A., Zoppou, C., Nielsen, O., Day, S., \& Roberts, S. (2004). Quantifying social vulnerability: a methodology for identifying those at risk to natural hazards. Australia: Geoscience Australia Record. 
Geng, H., Chen, F., \& Wang, Q. (2010). Countermeasures about how to improve the validity of SEA of overall coal mine district plan. China Coal, 36(3), 18-22 (in Chinese).

Li, G., \& Zhou, W. (2006). Impact of karst water on coal mining in North China. Environmental Geology, 49, 449-457.

Moran, J., Granada, E., Miguez, J. L., \& Porteiro, J. (2006). Use of grey relational analysis to assesses and optimize small biomass boilers. Fuel Processing Technology, 87(2), 123-127.

National Bureau of Statistics of China. (2012). China statistical yearbook 2011. Beijing: China Statistics Press.

Si, H., Bi, H., Li, X., \& Yang, C. (2010). Environmental evaluation for sustainable development of coal mining in Qijiang, western China. International Journal of Coal Geology, 81, 163168.

Song, D., Qin, Y., Zhang, J., \& Zheng, C. (2007). Concentration and distribution of trace elements in some coals from northern China. International Journal of Coal Geology, 69, 179191.

Sun, G. (2010). Coal in China: resources, uses, and advanced coal technologies. Coal Initiative Reports, 3, 1-26.
Svoray, T., \& Ben-Said, S. (2010). Soil loss, water ponding and sediment deposition variations as a consequence of rainfall intensity and land use: a multi-criteria analysis. Earth Surface Processes and Landforms, 35, 202-216.

Tyra, M. A., Groppo, J. G., Robl, T., \& Minster, T. (2003). Using digital mapping techniques to evaluate beneficiation potential in a coal ash pond. International Journal of Coal Geology, 54, 261-268.

Wang, C., \& Si, Q. (2011). A study of data statistical processing method of Delphi method and its application. Journal of Inner Mongolia Finance and Economics College, 9(4), 92-96.

Xue, Q., Liang, B., Wang, H., \& Liu, L. (2006). Numerical simulation of trace element transport on subsurface environment pollution in coal mine spoil. Journal of Trace Elements in Medicine and Biology, 20, 97-104.

Zhang, H., Song, J., Su, C., \& He, M. (2013). Human attitudes in environmental management: fuzzy cognitive maps and policy option simulations analysis for a coal-mine ecosystem in China. Journal of Environmental Management, 115, 227234. 